\title{
Integration of Human Intent Model Descriptions in Product Models
}

\author{
László Horváth \\ Budapest Polytechnic, John von Neumann Faculty of Informatics \\ e-mail:lhorvath@zeus.banki.hu \\ Imre J. Rudas \\ Budapest Polytechnic, John von Neumann Faculty of Informatics \\ e-mail:rudas@zeus.banki.hu \\ Carlos Couto \\ University of Minho \\ ccouto@dei.uminho.pt
}

Abstract: Advanced product model based style of engineering design together with internet based globalized project work relies upon advanced communication of model data. Model entities and their parameters represent decisions of engineers. Present day models of mechanical systems do not contain data about the background of human decisions. This situation motivated the authors at their investigations on exchange design intent information between engineers. Their concept was extending of product models to be capable to describe design intent information. Several human-computer and humanhuman communication issues were also considered. The complex communication problem has been divided into four sub-problems, namely communication of human intent source with the computer system, representation of human intent, exchange of intent data between modeling procedures and communication of the represented intent with humans. The paper is structured as follows. Firstly, main objectives of the research, earlier results and an approach to describe design intent in product models are outlined. Following this, utilization of human-computer procedures in 
modeling of design intent is characterized. Then main structure of model description of design intent is detailed. Finally design intent description in product modeling environment, is discussed and illustrated by examples.

Key words: Product modeling, Modeling of design intent, Model representation, Humancomputer interaction.

\section{INTRODUCTION}

Engineering workplaces within a work group are situated in different geographical sites. Advanced, customer oriented product design involves frequent revision of decisions. Modification of a decision also requires modification of related decisions. A prerequisite of this activity is availability of information on the background of the original decision. This is why modification of an original decision requires contribution of the original decision maker or an other authorized engineer. The authors investigate application of computer description of design intent to replace original decision maker in product modeling. They proposed a computer method to assists the modification of decisions by modeling of design intent.

In advanced CAD/CAM systems successful efforts resulted excellent software tools for description of relationships between entities within the product model [3]. Logical continuation of these initial steps is mapping intent description entities to product model entities. This is the main content of the work presented in this paper. The authors investigated computer representation of design intent in advanced modeling systems during their earlier works [1]. They revealed characteristics and description of design intent [2]. Instead of individually created experimental environment, they decided to utilize commercial modeling systems that contain open surfaces for development tasks similar to intent modeling.

The paper is structured as follows. Firstly, main objectives of the research, earlier results and an approach to describe design intent in product models are outlined. Following this, utilization of human-computer procedures in modeling of design intent is characterized. Then main structure of model description of design intent is detailed. Finally design intent description in product modeling environment, is discussed and illustrated by examples.

\section{AN APPROACH TO DESCRIPTION OF DESIGN INTENT IN PRODUCT MODELS}

Design intent modeling is suitable for description and handling of all information and knowledge in the background of product related decisions. 
A modeling system with engineering workplaces situated in different geographical sites was considered. A process parallel with product modeling has been established for modeling of design intent.

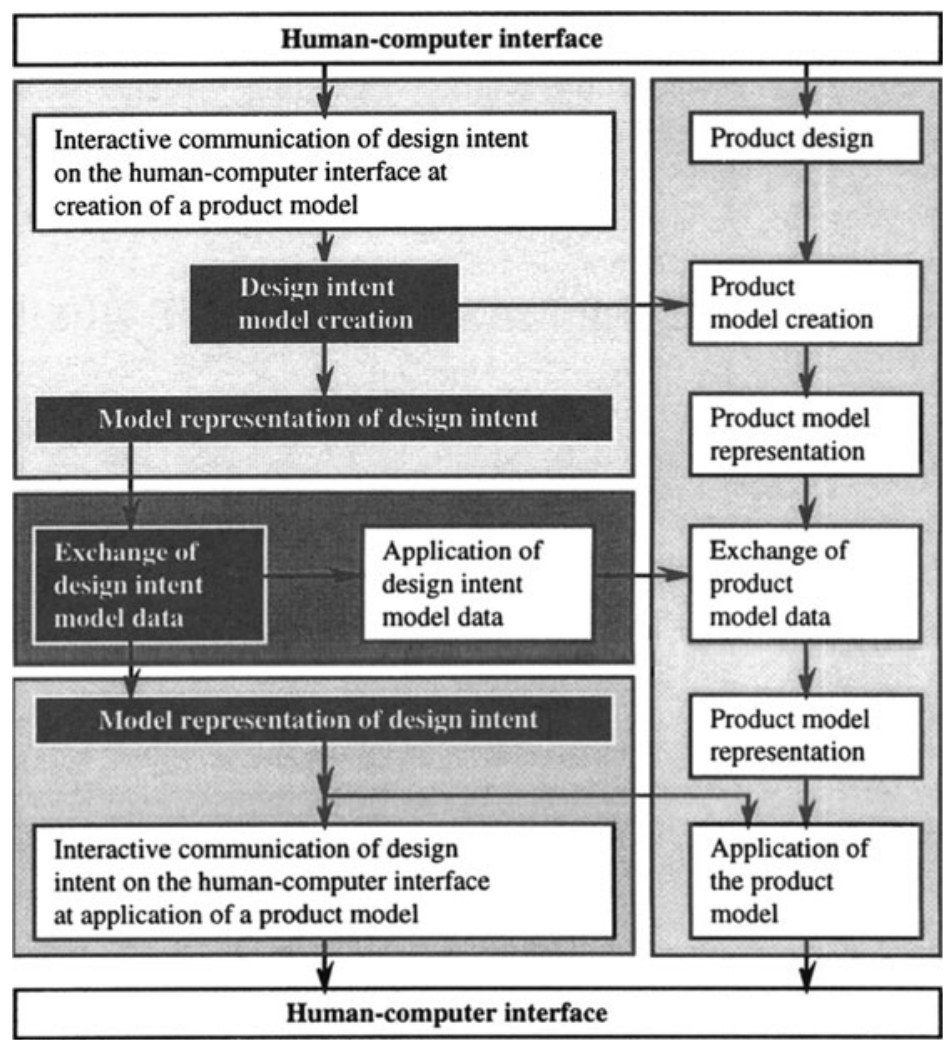

Fig. 1 Creating and processing of design intent

Creating and processing of design intent are outlined and related to normal product modeling activities in Fig. 1. Intent entities are created during product modeling in close connection with engineering decisions for product design. These entities are represented and related to results of the product design process then used by other product modeling and product model application procedures as well as humans. Modeling systems in the practice have various model representation capabilities and sets of entities. In the course of model data communication between different modeling systems, model data often should be translated and converted. This conversion is assisted by design intent information. Reference modeling and standard model description languages as EXPRESS make it possible to achieve integration of intent models into product model. 
Four sub-problems have been identified in modeling of design intent. The first is gathering intent information in the course of interactive graphic communication between human and computer at creation of a model. The second is representing design intent information in a model and its mapping to other product model entities. The third is communication of intent model entities between concurrent engineering modeling systems of different representation capabilities. The fourth is how intent description can be communicated with an engineer on graphic user interface at an application of a model.

\section{HUMAN-COMPUTER PROCEDURES AND DESIGN INTENT}

Procedures for human-computer interaction (HCI) are being adapted and enhanced for effective communication based design intent modeling. Modeling of design intent utilizes knowledge acquisition, data access and authority control, functions for human interactions as well as analyses in human behavior, human-human communication and human error (Fig. 2).

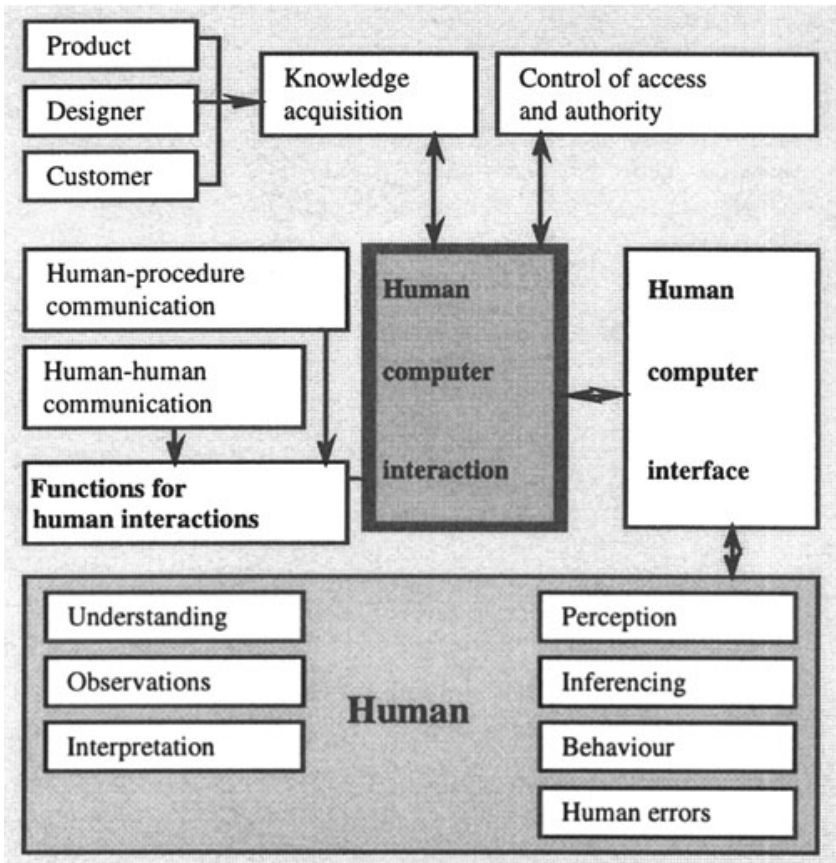

Fig. 2 Human related issues 
Design intent often can be defined in some form of knowledge. The related knowledge is not only domain but also product, designer and customer related. Consequently, it can not be involved in the modeling system in one of the conventional ways. Members of a group of engineers who are working on a product design can describe or point to different knowledge sources. Sometimes knowledge is of personal nature. In other cases, access to given knowledge sources is allowed for several engineers in a work group.

Quality of an engineering decision depends on the performance of the human decision maker. This performance can be increased effectively by using of computer based decision assistance. At the beginning of her or his career an engineer is not well trained. Computer description of intents of skilled engineers can support beginners at their decision making. Computer can learn decisions of skilled and experienced engineers in order to support decisions of less skilled engineers. Moreover a threshold knowledge can be described and then used for the purpose of excluding untrained or careless engineers from decision making and avoiding fatal errors. This approach is cited as mutual adaptive human-computer interfacing [4].

A design intent often is based on observations. Design or planning tasks are solved during cognitive processes where results of decisions are found out by designers. In similar cases it is very difficult to describe the human thinking process. Sometimes variants for types, parameter value ranges or discrete parameter values can be defined in intent descriptions. The authors considered modeling of design intent for model variants besides the a single decided variant. This makes it easier to change the decision to a more appropriate one during the application of the original product model. Understanding a given situation can be improved by appropriate intent descriptions from other engineers. This is the case of human-human communication using intent description.

Equally important is the role of design intent description at prevention, detection, diagnose and correction of human and processing errors. Error related behaviors of humans seriously affect their decisions [6]. Creating and handling of design intent are determined by cognitive behavior of humans. Perception and inference functions are equally essential at reasoning based decision making of a human. Improper design of a humanmachine system is often resulted in erroneous human activities [5]. A correct model of functions of humans who are in an interaction with computer procedures helps in understanding human-computer procedures. On the other hand role of computer generated suggestions in product design is important in avoiding errors. Engineers communicate computer systems through advanced graphic interface systems [7]. 


\section{MODEL DESCRIPTION OF DESIGN INTENT}

Entity parameters affected by an engineer decision can be defined as constraints and it can be related by constraints to other parameters of other entities. The proposed design intent modeling method offers different ways for definition of an intent at creating of a product model entity. Besides simple recording of intent information in intent model entities an intent creation procedure can work in close connection with the model creating procedure (Fig. 3). As an active way of intent creation, designer can be asked for intent information concerning a current decision. Similarly to the case of product model entity creation, authors suggest the application of the feature based concept for creating, relating and mapping intent model features. Intent feature libraries can be attached to product model feature libraries.

Easy to access background information packages can be linked to product model entities rather than involving them in model data structures by the application of Internet and related technologies. Large intent description data sets can be replaced by simple links in this manner. Remote sources of intents are maintained and actualized by engineers who created them.

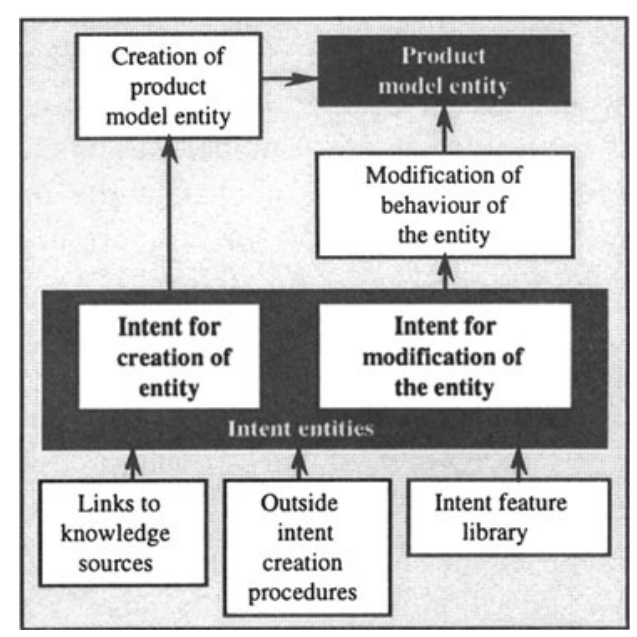

Fig. 3 Product model and intent entities

Remote access to background information is of outstanding importance when the applier of an intent is not authorized to use the knowledge source. Security measures are needed when running of a remote program is initiated at the application of the model. Industrial engineering practice produces 
very complex situations. As an example, members of a group of engineers can work on several design tasks for different companies. This style of work of engineers as well as the legislation background are to be taken into account at handling of design intent. An intent can be a simple identification of the decision maker such as the management of a company or an authority without any explanation.

Intent description is often created in the form of history of the background of a decision by using of a chain of intent entities. Fig. 4 shows a typical chain of design intent entities and several referred entities from the outside world. List of referred entities acts in the intent description as information source needed at processing of the intent description. Referred entities are accessed using links included in the intent description. History is considered as a chain of explanations for stages of a decision. Generic product models can involve generic intent models.

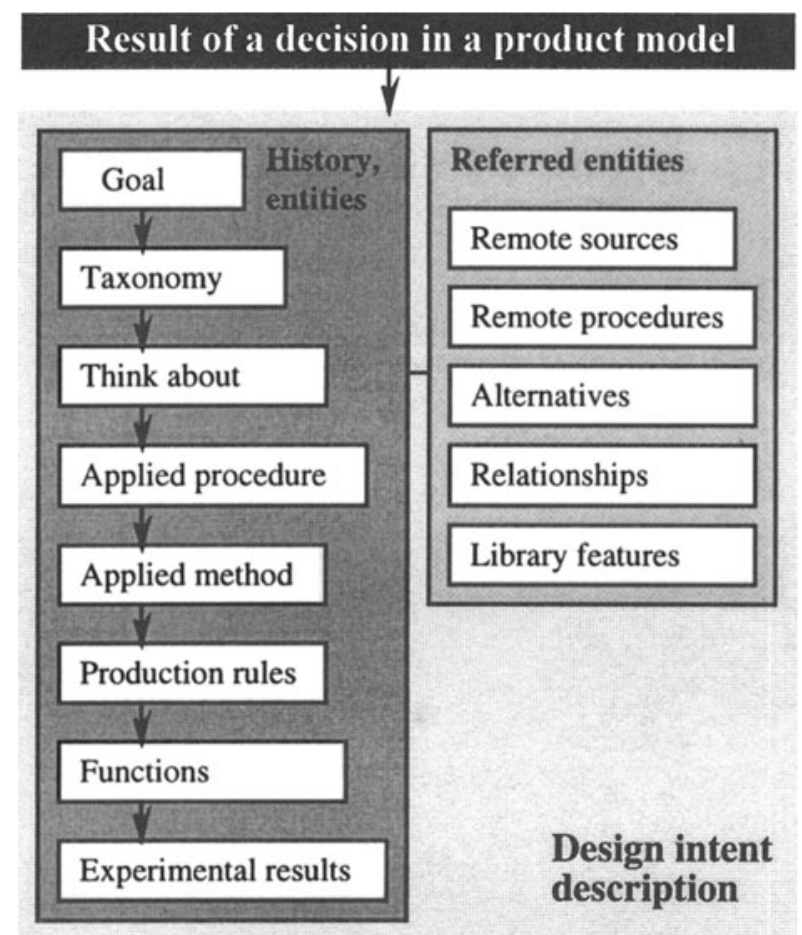

Fig. 4 Structure of a design intent

In the case of the example of Fig. 4 firstly a goal is defined for the decision then the related taxonomy is revealed. This is followed by a consideration of the applied procedure as a thinking process of the engineer. 
In other words she or he does not apply any idea from handbooks, etc. Next, the applied method is selected taking into consideration of the choice that is offered by the selected procedure. Alternative procedures and methods can be involved or referred if necessary. The procedure needs input data that were defined using production rules, functions and experimental results. The origin of the experimental results is an important element of the intent description. Fig. 4 shows only one of the possible styles of history.

\section{INTENT DESCRIPTION IN PRODUCT MODELING ENVIRONMENTS}

The authors assumed utilization of inherent intent description capabilities of open architecture object oriented concurrent engineering systems as a starting point for their work. Concurrent engineering systems with professional software modeling tools serve human-computer communication, creating, storage and multiple access of models, and application of models for further development, analysis, production planning and demonstration. Open surfaces of object oriented concurrent engineering systems are used to access modeling procedures, model data and graphic user interface through application programming interfaces (API).

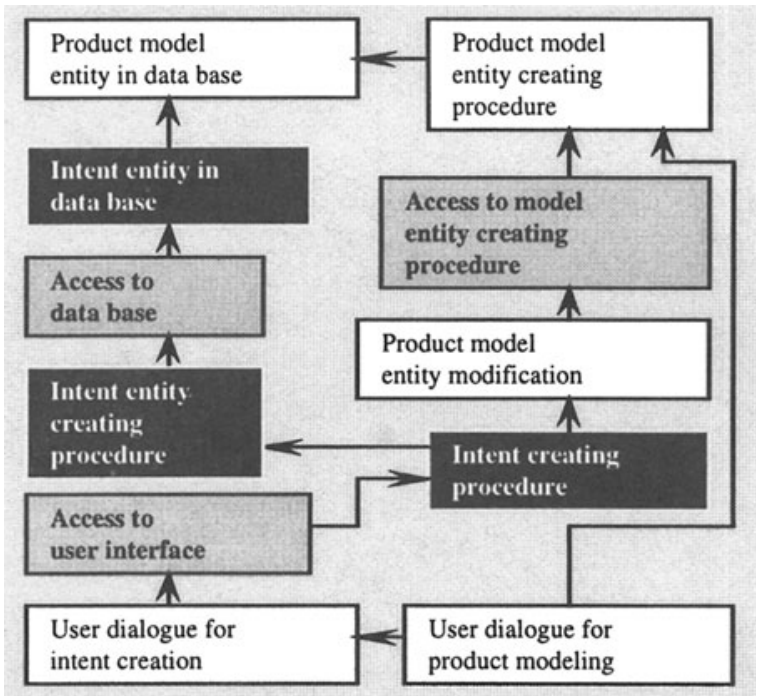

Fig. 5 Intent handling in an open architecture modeling system

A sketch of implementation of the proposed intent modeling is given in Fig. 5. User dialogue for product modeling is completed with dialogue for 
intent creation. Intent creation can be initiated automatically on the basis of the just defined model data. For example a computer procedure may ask the human that: "You defined an entity or its parameter. Describe why is it your decision and what to do if this decision should be changed by other engineers". In other cases intent creation is initiated by an engineer who describes: "This is why I decided as I did. If somebody should make some changes on this decision do the following..."

Access to user interface open system functions is necessary to establish communication between engineers and the intent creation procedures. The just defined intent entity can be used for modification of work of product model creation procedures. This is an active application of intent description. Active role of an intent can also be programmed for applications of product models. On the other hand engineers utilize intent descriptions at applications of product models. This is a passive application of intent model. Moreover, intent description can serve as a measure or even a command. Product model creating procedures and data bases are accessed from intent model handling procedures by open access functions of industrial modeling systems.

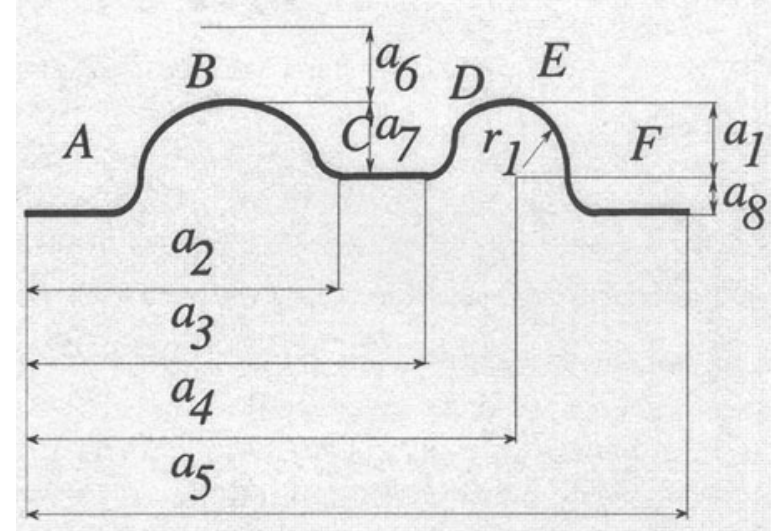

Fig. 6 Design intent at the creation of a section for creating a complex surface

Several typical considerations at modeling of design intent in the practice of modeling of mechanical parts are illustrated and discussed below through examples.

Complex surfaces are often created using complex curves that govern their shape. Fig. 6 shows a section of a complex surface that consists of six component surfaces $(A-F)$. Structural requirements were considered then surface types and dimensions were decided. Relationships of dimensions 
were defined then values of some dimensions were calculated. Fixed values of dimensions were defined as constraints. Other dimensions are allowed to be modified within well-defined ranges during applications of the model. These ranges were not described in the part model. Instead, one of the possible values was defined as a constraint. To assist later modification of the related decisions and to prevent later changes of the dimensions to an illegal range, the allowed ranges of dimensions were recorded as design intent entities. Component surfaces $A$ and $F$ should be flat and component surface $E$ should be cylindrical. All component surfaces are described using rational B-spline functions that offer free modification of their shape. Consequently, type of $A, E$ and $F$ surfaces should be constrained to avoid their modification as free form surfaces. There is a relationship between dimensions $A 4$ and $R l$. Elements of the intent description for the above discussed example are:

- Decision on relationship $A_{4}-R_{1}$. This decision is based on a method by an expert. Several alternative solutions were defined. They were involved in the intent model.

- Shape of component surfaces $B$ and $D$. Modifications of these free form surfaces are allowed. Although dimensions $A_{1} \min$ and $A 6$ limit the modification of these surface components.

- Shape of flat component surface $C$. This surface can be modified as a free form surface if mating surfaces of other parts are modified accordingly. Cost consequences can be estimated by a procedure that is related to the intent description and can be accessed through Internet.

- Fillet surfaces connecting component surfaces can be modified. Continuity between the connected surfaces must be maintained.

Description of design intent using the method proposed by the authors assists at the task of styling of the above detailed surface complex without changes that are not allowed by mechanical and manufacturing engineering. Styling engineer may create additional intent entities for additional styling and manufacturing planning procedures. A styled surface often produces problems or excess cost at manufacturing. A minor modification of the surface may result in cutting of the manufacturing cost dramatically. For this purpose design intent may involve description of allowable ranges of control point positions.

Fig. 7 illustrates basic relationships within and between parts of a mechanical assembly. The parameter model in the model of the part 1 includes the relationship $A 2=3,1 \mathrm{Al}$. The intent model includes a method that was used for creation of this formula. Sometimes parameters from the application environment determine parameters of some entities in the product design stage. At the same time quality of a design may be 
deteriorated by modifications that seem logical but contradict to the purpose of the product. Design intent description is essential in these cases and can be utilized at effective modification of the design. Engineers considered dimensions $A 3$ and $A 4$ as limited only by the contact constraints defined at the faces $F 1$ and F2. An other limiting factor can be the minimum allowed value of the gap between part 1 and part 2 .

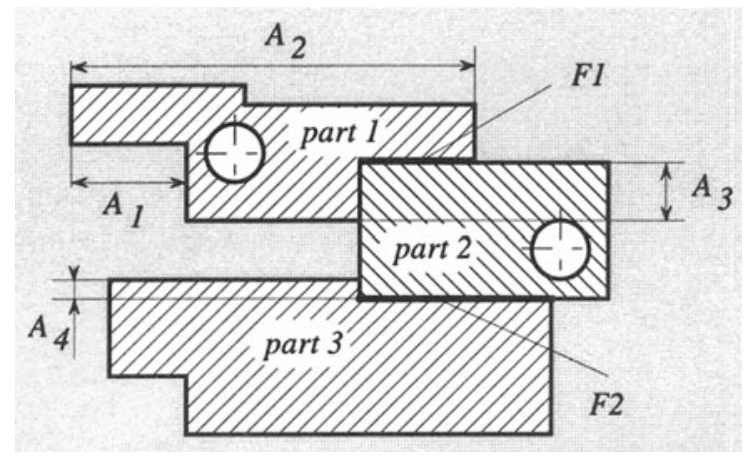

Fig. 7 Mechanical assembly

Is an intent count for much? Similar questions affect application of intent information. The answer is often subjective. Fortunately, decisions can be assisted by the description of origin, limits, strength and consequence of omitting of an intent. On the other side, engineers sometimes are not able to produce a good explanation for their decision. This is because intuition and anticipation are still important factors at decisions of skilled and experienced engineers. An intent description acts on behalf of the engineer who created it.

\section{CONCLUSIONS}

The authors proposed a method for modeling of the background of decisions using design intent descriptions. They proposed a method for mapping design intent model entities to product model entities. Intent modeling describes human thinking process and considerations behind human decisions. Intent description can be used by both model application processes and humans who handle them. Implementation of the proposed method assumes object oriented industrial modeling systems with open surface. Intent entities referred from the outside world are applied to link intent information to intent model that are not economical or not allowed to involve in the intent models. The proposed method can be considered as an extension of collaborative engineering. Outstanding importance of its 
application is where the original decision makers are not accessible and the only assistance at evaluation or modification of an earlier decision is the application of design intent description. Intent modeling is not modeling of what should be done by an engineer but what did an engineer and what are consequences of possible future work of other engineers. It can be considered as a well-prepared substitution of engineers by computer procedures.

\section{ACKNOWLEDGMENTS}

The authors gratefully acknowledge the grant provided by the OTKA Fund for Research of the Hungarian Government. Project number is $T$ 026090. Authors also would like thank Hungarian-Portugal Intergovernmental Science \& Technology Cooperation Programme for the financial support.

\section{REFERENCES}

[1] László Horváth, Imre J. Rudas: Attaching Knowledge to Product Model for Representation of Human Intent, Proceedings of the 1997 IEEE International Conference on Systems, Man and Cybernetics, Computational Cybernetics and Simulation, Volume 2, Orlando, Florida, USA, 1997, pp. 1580-1585.

[2] László Horváth, Imre J. Rudas: Representation of Human Intent in Computer Aided Engineering Design, Proceedings of the Second IEEE International Conference on Intelligent Processing Systems, Gold Coast, Australia, 1998 , pp 424-428.

[3] Kimura, F. - Suzuki, H.: A CAD System for Efficient Product Design Based on Design Intent, Annals of the CIRP, Vol. 38/1, 1989, pp:149-152.

[4] Yoshikawa, H. - Takahashi, M.: Conceptual Design of Mutual Adaptive Interface. Preprint of Integrated Systems Engineering Conference, Baden-Baden, 1994, pp. 221 226.

[5] Jose M. Nieves, Andrew P. Sage: Human and Organizational Error as a Basis for Process Reenginering: With Applications to Systems Integration Planning and Marketing, IEEE transactions on Systems, man and Cybernetics Part A: Systems and Humans, Volume 28, No. 6, 1998, pp 742-762.

[6] Pietro Carlo Cacciabue: A Methodology of Human Factors Analysis for Systems Engineering: Theory and Applications, IEEE transactions on Systems, man and Cybernetics Part A: Systems and Humans, Volume 27, No. 3, 1997, pp 325-339.

[7] L. J. Haasbroek: Advanced Human-Computer Interfaces ant Intent Support: A Survey and Perspective, 1993, IEEE International Conference on Systems, Man and Cybernetics, Lille, 1993, pp. 350-355. 